\title{
Antiferromagnetic ordering of magnetic moments due to cavity trapped electrons in sodium electrosodalite: A zero-field $\mu$ SR study
}

\author{
R. Scheuermann* and E. Roduner \\ Institute of Physical Chemistry, University of Stuttgart, Pfaffenwaldring 55, D-70569 Stuttgart, Germany \\ G. Engelhardt \\ Institute of Chemical Technology, University of Stuttgart, Pfaffenwaldring 55, D-70569 Stuttgart, Germany
}

H.-H. Klauss

Institute of Metal Physics and Nuclear Solid State Physics, Technical University of Braunschweig, Mendelssohnstraße 3, D-38106 Braunschweig, Germany

D. Herlach

Laboratory for Muon-Spin Spectroscopy, Paul Scherrer Institut, CH-5232 Villigen PSI, Switzerland

(Received 19 April 2002; published 31 October 2002)

\begin{abstract}
The long-range magnetic ordering of $\mathrm{Na}_{4}{ }^{3+}$ clusters with spin $S=1 / 2$ on a body-centered-cubic lattice in sodium electrosodalite has been studied in zero applied magnetic field by positive muons as local magnetic probes. An experimental determination of the temperature dependence of the magnetic order parameter of an $s$-electron antiferromagnet is presented. The order parameter is measured via the local magnetic field at the muon site $\left(B_{\text {loc }}\right)$. The temperature dependence $B_{\text {loc }}(T)$ exhibits critical behavior near the Néel temperature $T_{\mathrm{N}}=(50.3 \pm 0.2) \mathrm{K}$. The critical exponent $\beta=(0.36 \pm 0.1)$ is close to the predicted value for a threedimensional Heisenberg system. The precession signal is ascribed to muons in a diamagnetic state. A fraction of muons not contributing to the precession signal below $T_{\mathrm{N}}$ is ascribed partly to muons in domains which are not magnetically ordered and partly to muons forming a bound state with an unpaired electron.
\end{abstract}

DOI: 10.1103/PhysRevB.66.144429

PACS number(s): 75.50.Ee, 75.40.Cx

\section{INTRODUCTION}

Zeolites are crystalline nanoporous alumosilicates with a wide variety of structures and compositions. The main interest lies in their utility for adsorption and molecular separation processes and, as a consequence of their pore geometry, as shape-selective catalysts. ${ }^{1}$ Furthermore, their periodic arrangement of pores and cages has always been fascinating as a base structure that should permit the manufacturing of regular arrays of nanosized clusters with tailored properties - for example, for information technology.

The sodalite structure is the simplest zeolite structure with a framework built by an alternating network of cornersharing $\mathrm{AlO}_{4}$ and $\mathrm{SiO}_{4}$ tetrahedra, forming truncated octahedral cages ( $\beta$ or sodalite cages) ordered in a body-centeredcubic lattice (Fig. 1). The unit cell contains two $\beta$ cages joined through single four- and six-ring windows. In anhydrous sodium sodalite three sodium cations inside each $\beta$ cage compensate for the negative charge of the framework, leading to the chemical composition $\left[\mathrm{Na}_{3}\right]_{2}\left[\mathrm{Si}_{6} \mathrm{Al}_{6} \mathrm{O}_{24}\right]$ per unit cell. When exposed to sodium vapor at elevated temperatures, one excess sodium atom per cage is absorbed and ionized, forming a $\left[\left(\mathrm{Na}^{+}\right)_{4} \mathrm{e}^{-}\right] \equiv \mathrm{Na}_{4}{ }^{3+}$ ionic cluster (four equivalent alkali cations in a tetrahedral geometry sharing one electron). ${ }^{2}$ This type of defect is commonly referred to as the $F$ center, adopted from ionic solids where it denotes an anionic vacancy binding one electron. An increasing concentration of such $F$ centers changes the color from white to blue, purple, and finally to black, leading to the notation "black sodalite" for highly sodium doped samples. Such sys- tems can also be regarded as the mineral sodalite where central cage anions are replaced by electrons. They are therefore named sodium electrosodalite (SES), following the notation used in Ref. 3.

In the case of SES an almost complete loading with an $F$-center density of $1.4 \times 10^{21} \mathrm{~cm}^{-3}$ can be obtained, making it a unique model system to study interactions between $F$ centers arranged on a bcc lattice with a lattice constant of $8.875 \AA$. In most magnetic oxides the unpaired electrons

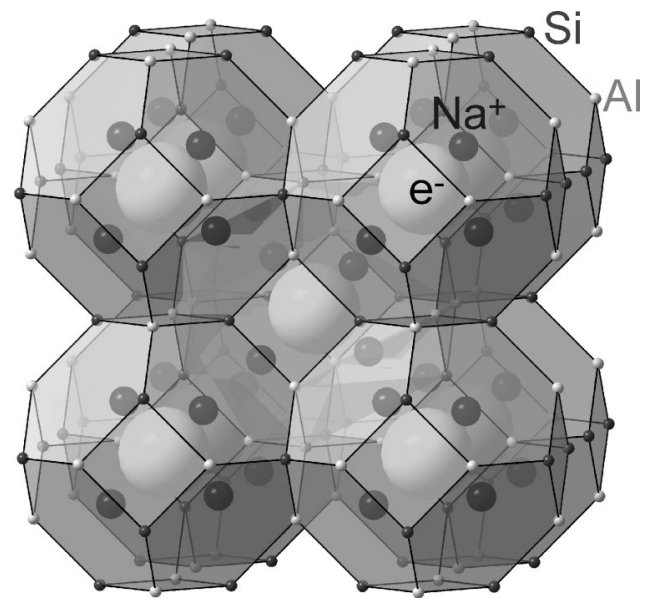

FIG. 1. Body-centered-cubic lattice of $F$ centers in a sodalite matrix. Four sodium ions arranged tetrahedrally inside a $\beta$ cage share one electron. The framework is built of corner-sharing $\mathrm{AlO}_{4}$ and $\mathrm{SiO}_{4}$ tetrahedra; the oxygen atoms between the $\mathrm{Al}$ and $\mathrm{Si}$ atoms are omitted for clarity. 
occupy $d$ or $f$ orbitals of transition-metal or rare-earth atoms. In others, such as organic magnets, they possess the low symmetry of some often quite extended molecular orbitals. Therefore, SES is an attractive compound for model studies of magnetic interactions: the electrons occupy sites of tetrahedral symmetry of mostly $s$ character, ${ }^{4}$ imposed by the coordination to the four equivalent sodium ions.

An antiferromagnetic phase transition in SES with a Néel temperature $T_{\mathrm{N}}=48(2) \mathrm{K}$ was deduced from the temperature dependence of the paramagnetic shift of ${ }^{27} \mathrm{Al}$ in magic angle spinning nuclear magnetic resonance (MAS NMR), dc magnetic susceptibility measurements, and electron paramagnetic resonance studies. ${ }^{3}$ A Curie-Weiss behavior for the magnetic susceptibility was found at temperatures above 60 $\mathrm{K}$, with a Weiss temperature in the range $\Theta \approx-178(8) \ldots$ -210 (5) $\mathrm{K}$ (depending on the experimental technique used). Subsequent NMR studies ${ }^{5}$ verified these findings, yielding values $T_{\mathrm{N}}=54(2) \mathrm{K}$ and $\Theta=-168(5) \mathrm{K}$.

The $\mathrm{Na}^{+}$ions are located close to four out of the eight six-ring windows present in the sodalite cage. The four remaining six-ring windows are coordinated to sodium ions in the neighboring cages. As a consequence the tetrahedral electron wave functions have no shared corners, a fact that to a large extent suppresses a direct exchange interaction (the corner of each tetrahedron points towards the triangular base of a neighboring tetrahedron). There is also no direct exchange interaction to the nearest neighbor through the four-ring windows along the edge of the unit cell. Therefore, a superexchange mechanism via the cage atoms is responsible for the antiferromagnetic ordering. Local spin density approximation (LSDA) band-structure calculations showed an energy gap between valence band and conduction band with a magnitude about $0.1 \mathrm{eV}$, meaning that SES is a Mott semiconductor. ${ }^{6,7}$ The ground state of a bcc lattice of bare $\mathrm{Na}_{4}{ }^{3+}$ clusters is metallic and diamagnetic whereas in the presence of the aluminosilicate framework an antiferromagnetic semiconducting state is lowest in energy. ${ }^{6}$ The nearestneighbor coupling constants $J_{n n}$ turned out to be much stronger than the next-nearest-neighbor couplings $J_{n n n},{ }^{7,8}$ resulting in an antiferromagnetic bcc structure of two simple cubic sublattices. $J_{n n} / k_{\mathrm{B}}$ corresponds to a critical temperature of about $50 \mathrm{~K}$.

In the present work zero-field muon spin rotation $(\mu \mathrm{SR})$ experiments have been performed to study the long-range magnetic ordering of the $F$ centers in SES which is reflected in the onset of a precession signal with decreasing temperature. From the secondary beamlines $\pi \mathrm{E} 3$ and $\pi \mathrm{M} 3$ of the proton accelerator at the Paul Scherrer Institute (PSI, Villigen, Switzerland), a beam of almost $100 \%$-polarized muons is obtained. When implanted into a sample the muons maintain their spin polarization. Their further fate depends on the properties of the sample. In general, two different species have to be distinguished-namely, muons in paramagnetic states and others in diamagnetic states-depending on whether or not they are hyperfine coupled to an unpaired electron.

The time evolution of the muon-spin polarization $P(t)$ in both paramagnetic or diamagnetic states is governed by the magnetic interactions of the muon spin in the sample. With a mean lifetime of $\tau_{\mu}=2.197 \mu$ s the muon decays into two neutrinos and a positron. The angular distribution of the decay positron is anisotropic with respect to the muon spin at the moment of decay. $P(t)$ is obtained from measurements of the time between muon implantation and detection of its decay positron under a fixed direction for each individual muon and averaging over a muon ensemble of typically $10^{6}$ muons. The positron count rate in a detector is given by

$$
N(t)=N_{0} \exp \left(-\frac{t}{\tau_{\mu}}\right)[1-P(t)]+b g,
$$

where $N_{0}$ is a normalization constant and $b g$ is a timeindependent background.

The polarization function $P(t)$ in general is a superposition of several contributions, depending on how many different muon species are formed or how many different magnetic environments exist:

$$
P(t)=\sum_{i} a_{i} G_{i}(t)
$$

The so-called asymmetries $a_{i}$ are proportional to the polarization in a given muon state, and $G_{i}(t)$ denotes the specific relaxation functions. Calibration measurements $G_{1}(t) \equiv 1$ are usually performed on a silver sample, which yields the muon beam asymmetry $a_{1}=a_{\text {beam }}$ corresponding to $100 \%$ polarization. In the present work $a_{\text {beam }}=0.220(2)$.

A paramagnetic muon state, i.e., a bound state between the positive muon and an electron named muonium $(\mathrm{Mu})$, may be observed in insulators and semiconductors. The Fermi contact interaction gives rise to an effective hyperfine field at the muon site which determines the time evolution of the muon spin governed by the hyperfine Hamiltonian $H_{\mathrm{hf}}$ $=h \mathbf{S}_{u} \cdot \mathbf{A}_{\mu} \cdot \mathbf{S}_{e}$ (hyperfine tensor $\mathbf{A}_{\mu}=A_{\mu} \mathbf{1}, A_{\mu}$ in frequency units; $h$ denotes Planck's constant). The hyperfine coupling constant (hfc) $A_{\mu}$ is proportional to the electron-spin density at the muon. It seems plausible that a paramagnetic state can also be formed between a positive muon and the electron of a negatively charged $F$ center, simply based on the argument of Coulomb attraction. Such a state will further be denoted as $\mu^{+} @ F^{-}$.

The time evolution of the muon-spin polarization of a paramagnetic muon species in a longitudinal applied field is given by the sum of a nonprecessing and a precessing term, whose frequency is determined by the hyperfine coupling constant $A_{\mu}$ and the applied external magnetic field. In typical cases this frequency is too high to be observed experimentally. However, the easiest way to investigate the existence of such a paramagnetic species is in a longitudinally applied magnetic field. The coupling of the muon spin to the unpaired electron spin and to other spins leads to muon depolarization in low fields. High longitudinal magnetic fields decouple these interactions and lock the muon-spin direction so that the full polarization is recovered. The repolarization behavior is used to estimate the coupling constants and the fraction of muons involved. ${ }^{9}$

A diamagnetic muon state (in this work denoted as $\left.\mu^{+} @ d\right)$ is characterized by a zero net electron-spin density 


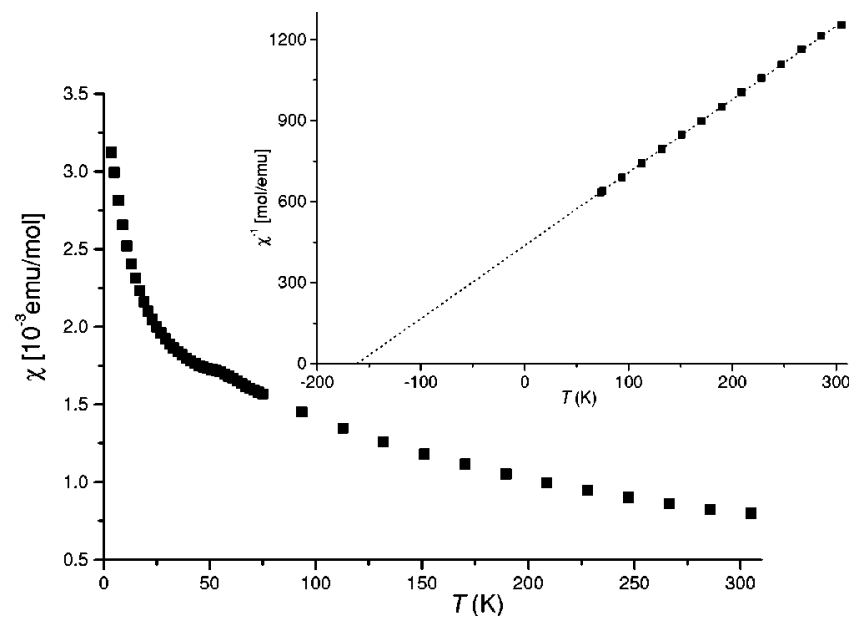

FIG. 2. Magnetic susceptibility of SES measured in $50000 \mathrm{G}$. The inset shows the result of a fit of $1 / \chi$ to a Curie-Weiss law in the paramagnetic phase. The antiferromagnetic ordering is indicated by a shoulder near $50 \mathrm{~K}$; the low-temperature upturn is assigned to impurity spins $\left(0.008 \mu_{\mathrm{B}}\right.$ per formula unit, determined by magnetization measurements at $2 \mathrm{~K}$ ).

at the muon site. Such states comprise bare positive muons or negatively charged muonium $\left(\mathrm{Mu}^{-}\right)$as well as muonium undergoing fast electron-spin exchange with an ensemble of unpolarized electrons, as in metals or in semiconductors at high temperatures. In this case, transverse magnetic field components with respect to the muon spin lead to Larmor precession and transverse field variations cause spin relaxation due to dephasing which is described by a relaxation function $G(t)$. For a static Gaussian field distribution, such as, for example, originating from a large number of dipole field sources, the relaxation function is also Gaussian. ${ }^{10}$ If the spin relaxation is due to a dipole-dipole interaction with dilute disordered magnetic moments (or, equivalently, magnetic holes in a magnetically ordered structure), where only a small perturbation of the local field occurs, its form is Lorentzian. ${ }^{11}$

For a spatially isotropic distribution of local fields in a sample, one-third of the muon spins does not experience a transverse field component and, hence, will not precess. However, relaxation will occur due to field fluctuations perpendicular to the initial muon-spin direction. Therefore, the corresponding polarization function consists of two terms: a damped precession signal plus a purely relaxing signal of half the amplitude of the precession signal.

\section{EXPERIMENTAL AND DATA ANALYSIS}

The powder sample of black sodalite studied in this work has been prepared as described in Ref. 3. The level of loading was determined by dc susceptibility $(\chi)$ measurements (Fig. 2) in a superconducting quantum interference device (SQUID) magnetometer. For a net composition $\mathrm{Na}_{4} \mathrm{Si}_{3} \mathrm{Al}_{3} \mathrm{O}_{12}$ the fit of the susceptibility to a Curie-Weiss law above $73 \mathrm{~K}$ yields a Weiss temperature $\Theta$ $=-162(1) \mathrm{K}$ and an effective magnetic moment of $\mu_{\text {eff }}$ $=1.71 \mu_{\mathrm{B}}$ per $\beta$ cage (Bohr magneton $\mu_{\mathrm{B}}=9.274$ $\times 10^{-24} \mathrm{~J} \mathrm{~T}^{-1}$ ). This corresponds to a loading level of almost 99\%. [For a fully loaded sample $\mu_{\text {eff }}$ $=g_{\mathrm{e}} \sqrt{S(S+1)} \mu_{\mathrm{B}}=1.73 \mu_{\mathrm{B}}$.] Allowing an offset of $\chi$ (high-temperature limit) the values change slightly to $\Theta$ $=-152(1) \mathrm{K}$ and $\mu_{\mathrm{eff}}=1.66 \mu_{\mathrm{B}}$ (corresponding to a loading level of $96 \%$ ).

In our experiment $280 \mathrm{mg}$ SES were sealed in a $10 \times 10$ $\times 1.5 \mathrm{~mm}^{3}$ polyethylene plastic bag under helium atmosphere and mounted in a helium flow cryostat of one of the PSI facility spectrometers (DOLLY in area $\pi \mathrm{E} 3$ and GPS in area $\pi \mathrm{M} 3$ ). The earth magnetic field was compensated to a residual value of less than $1 \mathrm{mG}$, and the temperature stability during the measurements was better than $0.01 \mathrm{~K}$. Analysis of the data was performed in the time domain, fitting the recorded histograms simultaneously to Eq. (3) by use of the least-squares method provided by the $\mu$ SR data analysis program MINFIT which is based on the MINUIT function minimization library. ${ }^{12}$

\section{RESULTS AND DISCUSSION}

\section{A. Muon states in the SES paramagnetic phase}

Transverse-field muon-spin rotation experiments have been performed above the Néel temperature in the temperature range $55-200 \mathrm{~K}$. At an applied field $B$ of 7, 100, 3000, or $6000 \mathrm{G}$ a single precession signal from $\mu^{+} @ d$ with a frequency $\nu_{\mu}=\gamma_{\mu} B\left(\gamma_{\mu}=13.55 \mathrm{MHz} / \mathrm{kG}\right.$ is the muon gyromagnetic ratio) was observed. The muon-spin polarization in this diamagnetic signal accounts for only about $70 \%$ of the full polarization, independent of temperature and magnetic field value.

Any paramagnetic muon species with a hyperfine coupling only between the muon and electron spins would lead to a precession signal with a frequency of about $10 \mathrm{MHz}$ at 7 $\mathrm{G}$, independent of the muon hyperfine coupling constant, which was not detected in the present experiment. Because in a semiconductor like SES the formation of a paramagnetic muon species would be expected, the decoupling method in a longitudinal field was applied in order to find the reason for the missing polarization. Despite the lack of suitable calibration measurements, which are essential for a quantitative analysis of such data, ${ }^{13}$ the increase of asymmetry with increasing longitudinal magnetic field (Fig. 3) clearly proves that a considerable fraction of muons form a paramagnetic species akin to muonium with a muon hfe of about 2000 MHz. Moreover, the steep increase in the low-field range up to $200 \mathrm{G}$ is typical for the decoupling of the muon spin from nuclear dipole moments, meaning that this is not a plain muon-electron bound state, but a system which is coupled to the spins of the surrounding nuclei $\left({ }^{23} \mathrm{Na},{ }^{27} \mathrm{Al},{ }^{29} \mathrm{Si}\right)$. This explains the absence of a precession signal in low transverse magnetic fields, where the nuclear hyperfine interaction (NHFI) leads to multiple line splitting with vanishing polarization in the individual signals. As outlined above it seems reasonable to assume this state to be a muon bound to the $F$ center of a $\beta$ cage $\left(\mu^{+} @ F^{-}\right)$. 


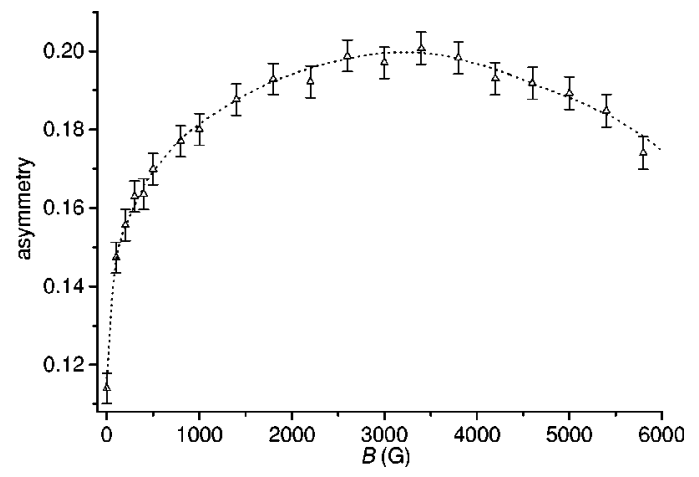

FIG. 3. Repolarization curve obtained at $50.4 \mathrm{~K}$ (paramagnetic phase); the dotted curve serves to guide the eye. The decrease of the asymmetry above $3500 \mathrm{G}$ is inherent to the spectrometer.

\section{B. Time dependence of the muon-spin polarization below $T_{N}$}

At temperatures below $50 \mathrm{~K}$ a precession signal shows up in the time dependence of the muon decay asymmetry $a(t)$ (Fig. 4). This demonstrates the onset of long-range magnetic ordering which leads to coherent muon-spin precession. The negative Weiss temperature obtained from SQUID measurements (Fig. 2) identifies the type of ordering as antiferromagnetic.

The data are analyzed using a polarization function $P(t)$ consisting of an exponentially damped oscillation and two terms describing pure relaxation (a slowly relaxing term of Gaussian line shape and a fast relaxing term of Lorentzian line shape). This three-component polarization function yields the best results in the temperature range 7-49.5 K:

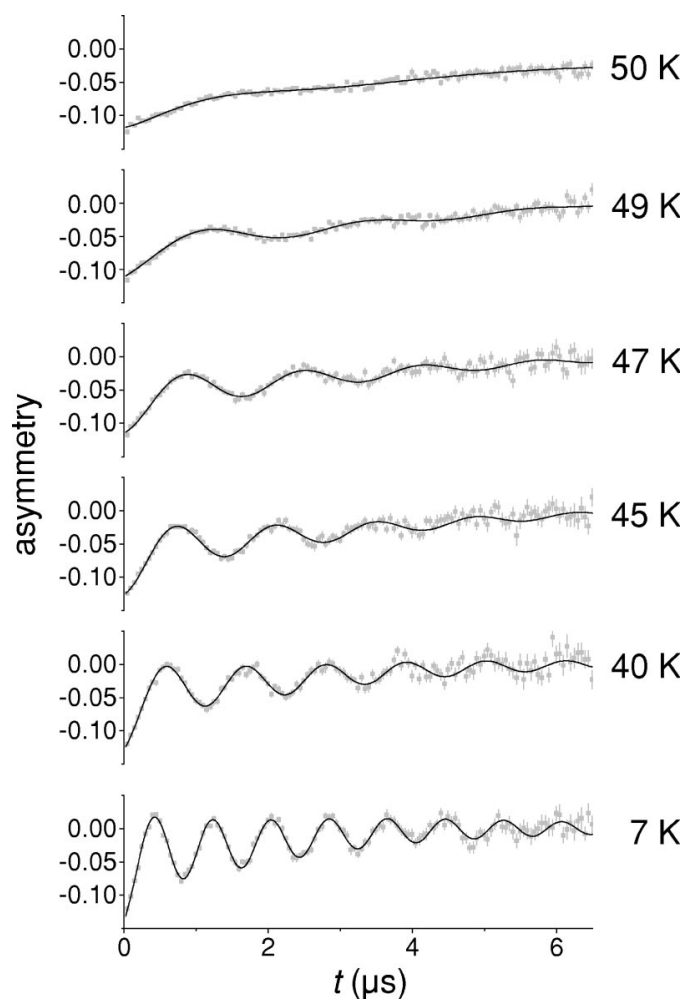

FIG. 4. Zero-field $\mu$ SR spectra of SES below the Néel temperature. The polarization was fitted to Eq. (3).

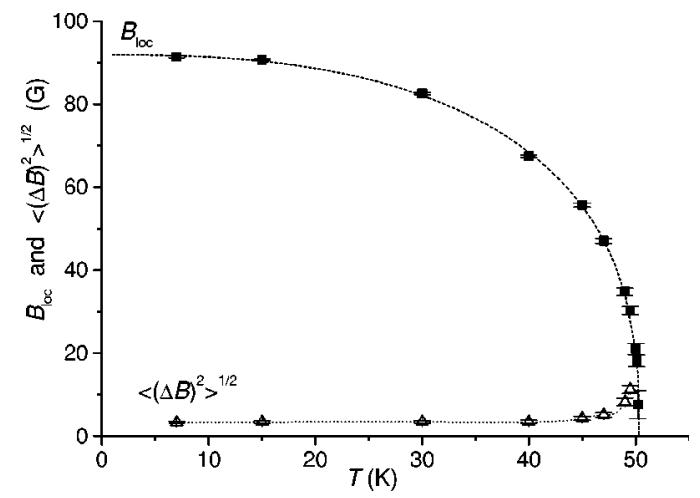

FIG. 5. Temperature dependence of the local magnetic field $B_{\text {loc }}$ (obtained from the precession frequency) and the static field inhomogeneity $\left\langle(\Delta B)^{2}\right\rangle^{1 / 2}$ (obtained from the damping rate $\lambda$ ) at the muon site for a diamagnetic muon species $\mu^{+} @ d$ in SES. The dashed line represents the results from a fit of $B_{\text {loc }}$ to Eq. (4); the dotted line serves to guide the eye.

$$
P(t)=a_{\mathrm{L}} \mathrm{e}^{-\lambda t} \cos \left(2 \pi \nu_{\mu} t-\varphi\right)+a_{\mathrm{G}} e^{-\sigma^{2} t^{2} / 2}+a_{\mathrm{F}} e^{-\Gamma t}
$$

An ansatz with a $2: 1$ ratio of the polarization contained in an oscillating and a purely relaxing signal $\left(G_{1}(t)\right.$ $=a_{1}\left[2 / 3 \exp (-\lambda t) \cos \left(2 \pi \nu_{\mu} t-\varphi\right)+1 / 3 \exp \left(-\lambda^{\prime} t\right]\right)$, which should be used in the case of a fully magnetically ordered powder sample with statistically oriented magnetic domains, results in significantly higher $\chi^{2}$ values. The main contribution (more than $90 \%$ at the lowest temperatures) to the Gaussian ralaxation signal corresponds to the $1 / 3$ signal fraction of muon spins experiencing the well-defined local field which leads to the precession signal. The exponential damping of the precession signal (relaxation rate $\lambda$ ) is typical for dilute disordered systems of perturbing magnetic moments ${ }^{11}$ which in the present case is realized by undoped $\beta$ cages (acting as "magnetic holes"), other magnetic impurities, or lattice distortions.

Above $49.5 \mathrm{~K}$, where the oscillation is rather weak, only the sum of an exponentially damped oscillation and a Gaussian relaxation is used. Due to the high correlation of the asymmetries and the relaxation rates, only the frequency $\nu_{\mu}$ could be determined reliably at these temperatures.

\section{Local magnetic field at the muon site}

The observed precession signal below $T_{\mathrm{N}}$ is attributed to a diamagnetic muon species $\mu^{+} @ d$. It cannot be due to the paramagnetic $\mu^{+} @ F^{-}$species since in this case the lowtemperature limit of the precession frequency would correspond to an internal field of approximately $1 \mathrm{G}$. In such a low field the NHFI of the surrounding nuclei of the sodalite framework $\left({ }^{27} \mathrm{Al},{ }^{23} \mathrm{Na}\right)$ would lead to unresolved multiple line splitting, thus preventing the observation of a single long-lived precession signal.

For $\mu^{+} @ d$ the local field at the muon site is obtained by $B_{\text {loc }}=\nu_{\mu} / \gamma_{\mu}$. The temperature dependence of $B_{\text {loc }}$ as a measure of the sublattice magnetization is displayed in Fig. 5. These data are fitted by a function which describes the critical behavior near the phase transition with a critical exponent 
$\beta$ and takes into account the suppression of the order parameter near $T=0$ by low-energy magnon excitations, phenomenologically represented by an additional exponent $\delta$ :

$$
B_{\text {loc }}(T)=B_{\text {loc }}(0)\left[1-\left(T / T_{\mathrm{N}}\right)^{\delta}\right]^{\beta} \text {. }
$$

From this fit the Néel temperature $T_{\mathrm{N}}=50.3(2) \mathrm{K}$ is obtained with a high accuracy. The critical exponent $\beta$ $=0.36(1)$ agrees with the theoretically predicted value of from $0.367(4)$ (Ref. 14) to $0.38(3)$ (Ref. 15) for a threedimensional Heisenberg system. $\delta=2.6(1)$ is in reasonable agreement with the theoretical value of 2 for a cubic sytem. ${ }^{16}$ In the low-temperature limit the local magnetic field at the muon site is $B_{\text {loc }}(0)=92.0(2) \mathrm{G}$. From the relaxation rate $\lambda$ the field inhomogeneity (width of the Lorentzian field distribution) is obtained via $\left\langle\left(\Delta B_{\text {loc }}\right)^{2}\right\rangle^{1 / 2}=\lambda /\left(2 \pi \gamma_{\mu}\right)$. Its value is only about $4 \%$ of $B_{\text {loc }}$ at $T=7 \mathrm{~K}$. The field inhomogeneity increases to a value of about $37 \%$ at $49.5 \mathrm{~K}$ and reaches the value of the local field (extrapolated value) at $49.9 \mathrm{~K}$ (Fig. 5 ). This reflects the breakdown of magnetic order near the phase transition.

The local magnetic field sensed by the muon is comprised of the dipole fields of localized magnetic moments (which vanish at lattice sites of cubic symmetry) and the hyperfine field (Fermi contact field) at the muon, which is given by the net electron-spin polarization. Calculations of the dipole field assuming an ordered magnetic moment of $1 \mu_{\mathrm{B}}$ ( $z$ component) at each cage center result in values which are too high to account for the observed precession frequency by at least a factor of 2 at any site in the lattice except near the centers of the six-ring windows of the $\beta$ cages, where the dipole field vanishes. One side of such a six-ring window possesses a coordinated sodium ion, the other side does not, and therefore the positive muon is likely to be pushed out of the window plane. Here the dipole field has a nonzero value. In addition a finite Fermi contact field may be present at this site. Alternative sites to host a muon in a locally diamagnetic environment are the doubly occupied nonbonding orbitals of the oxygen atoms of the aluminosilicate framework. However, at this site a Fermi contact field of approximately half the magnitude and opposite sign with respect to the dipole field is required to reduce the local field to the observed value.

\section{Coexistence of magnetically ordered and paramagnetic domains}

The temperature dependence of the asymmetries $a_{\mathrm{L}}, a_{\mathrm{G}}$, and $a_{\mathrm{F}}$ obtained from fits to Eq. (3) is shown in Fig. 6. For discussion, the polarization calculated from the asymmetries related to $a_{\text {beam }}$ is shown in Fig. 7 . The precession signal with asymmetry $a_{\mathrm{L}}$ plus a purely relaxing part $\left(a_{\mathrm{G}}\right)$ in the AFM phase is ascribed to muons in a locally diamagnetic environment. The ratio $a_{\mathrm{L}} / a_{\mathrm{G}}$ is less than 2 in the entire temperature range. The conclusion is that only part of $a_{\mathrm{G}}$ has to be attributed to $\mu^{+} @ d$ in magnetically ordered regions, whereas the remaining part is due to $\mu^{+} @ d$ in paramagnetic domains which even persist to the lowest temperatures measured. Thus, the volume fraction of antiferromagetic (AFM) domains is represented by $3 a_{\mathrm{L}} / 2$. With increasing temperature the AFM phase volume decreases by about $30 \%$. The
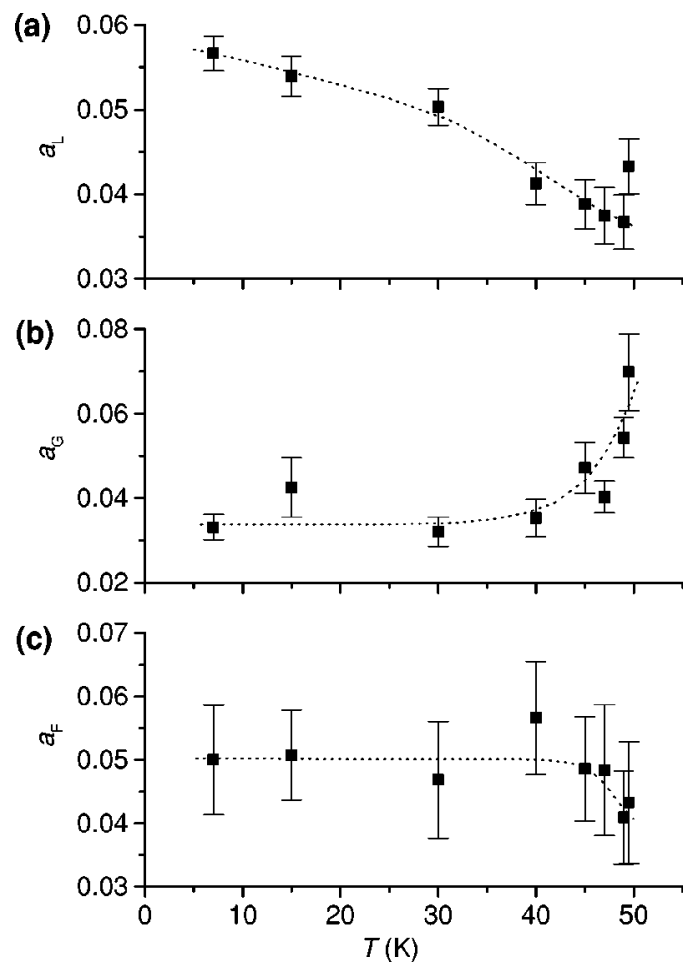

FIG. 6. Temperature dependence of the asymmetries obtained from a fit of the muon-spin polarization to Eq. (3). (a) Lorentzian component $a_{\mathrm{L}}$, (b) Gaussian component $a_{\mathrm{G}}$, and (c) fast relaxing component $a_{\mathrm{F}}$. The dashed lines are guides to the eye.

volume fraction of the paramagnetic domains below $40 \mathrm{~K}$ is given by the difference between the total diamagnetic signal fraction $\left(a_{\mathrm{L}}+a_{\mathrm{G}}\right)$ and $3 / 2$ times the precession signal $a_{\mathrm{L}}$ (see Fig. 7) and varies from less than $10 \%$ at $10 \mathrm{~K}$ to about $20 \%$ at $40 \mathrm{~K}$. Above $40 \mathrm{~K}$ the increase of the total diamagnetic signal amplitude $a_{\mathrm{L}}+a_{\mathrm{G}}$ is attributed to an additional channel of polarization transfer from the paramagnetic $\mu^{+} @ F^{-}$to the diamagnetic $\mu^{+} @ d$ species. As the individual components attributed to the Gaussian signal of the polarization cannot be separated, the relaxation rate $\sigma$ $\left(\approx 0.3-0.5 \mu \mathrm{s}^{-1}\right.$ below $\left.T_{\mathrm{N}}\right)$ is regarded as a phenomenological fit parameter only.

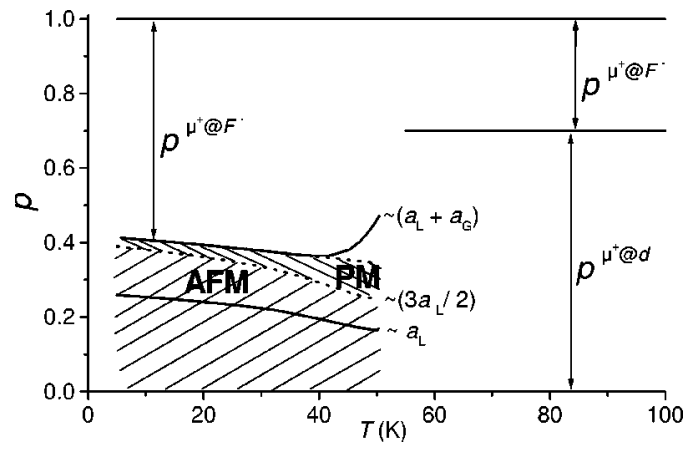

FIG. 7. Normalized polarization fractions $a_{\mathrm{L}, \mathrm{G}} / a_{\text {beam }}$ vs temperature. The volume fractions of the diamagnetic signal (antiferromagnetic, AFM; paramagnetic, PM) are indicated. 


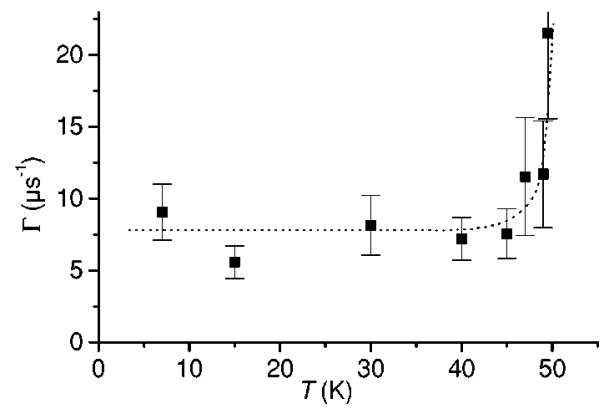
$\Gamma(T)$.

FIG. 8. Temperature dependence of the fast relaxation rate

\section{E. Paramagnetic center below $T_{\mathrm{N}}$}

The temperature dependence of the asymmetry $a_{\mathrm{F}}$ and the relaxation rate $\Gamma$ of the third term in Eq. (3) are shown in Figs. 6(c) and 8, respectively. This signal is attributed to the paramagnetic muon species $\mu^{+} @ F^{-}$which is detected in the longitudinal field repolarization experiments above $T_{\mathrm{N}}$ (cf. Sec. III A).

Only $1 / 2$ of the asymmetry of any paramagnetic muon state can be detected experimentally. This value may be further reduced by the NHFI and multiple line splitting in environments with transverse magnetic field components. Therefore, the effective asymmetry $\left(a_{\mathrm{L}}+a_{\mathrm{G}}+2 a_{\mathrm{F}}\right)$ accounts for $90 \%$ of the full muon-spin polarization.

At the lowest temperatures the formation probability of $\mu^{+} @ F^{-}$is about $60 \%$; with increasing temperature it decreases to about $50 \%$ at $49.5 \mathrm{~K}$ (Fig. 7). This decrease, along with the increasing relaxation rate $\Gamma$ (Fig. 8), suggests a transition of $\mu^{+} @ F^{-}$to a diamagnetic state, in accordance with the larger fraction of $\mu^{+} @ d$ above the Néel temperature.

\section{SUMMARY}

Fully loaded sodium electrosodalite represents a model system of a bcc lattice of ionic clusters, analogous to $F$ cen- ters in ionic solids. The long-range antiferromagnetic ordering of these clusters is detected in zero applied magnetic field by the onset of spontaneous muon-spin precession below the Néel temperature. The precession signal is attributed to muons in a diamagnetic state. The temperature dependence of the local magnetic field at the muon site, deduced from the precession frequency, follows the behavior of the sublattice magnetization of a three-dimensional Heisenberg system. This isotropic interaction is expected for the cubic system. From the magnitude of the calculated dipole field the most likely muon site is near the center of the six-ring windows of the $\beta$ cages. Other possible muon sites are the nonbonding orbitals of the oxygen atoms of the aluminosilicate framework. A coexistence of magnetically ordered and paramagnetic domains is deduced from a nonprecessing signal fraction significantly larger than the expected value for an isotropic field distribution and persists even at the lowest temperatures. Near the phase transition the breakdown of magnetic order shows up in an increasing inhomogeneity of the local field, along with a decrease of the volume fraction of antiferromagnetic domains. In addition, above $T_{\mathrm{N}}$ a paramagnetic muon state is identified by the field dependence of the muon-spin polarization. We assign this state to a positive muon bound to the negatively charged $F$ center.

\section{ACKNOWLEDGMENTS}

We thank the instrument scientists Dr. A. Amato and Dr. W. Waeber (PSI Laboratory for Muon Spin Spectroscopy) for their help with the experiments, Dr. A. Schenck (Institute of Particle Physics of ETH Zurich) for many helpful discussions, Th. Schmauke [Institute of Physical Chemistry, University of Stuttgart, SQUID measurements at the 3. Physical Institute of the University of Stuttgart (Professor M. Mehring)], and D. Menzel (Institute of Semiconductor Physics and Optics, Technical University of Braunschweig, SQUID measurements).
* Now at Paul Scherrer Institut, CH-5232 Villigen PSI, Switzerland.

${ }^{1}$ Introduction to Zeolite Science and Practice, edited by $\mathrm{H}$. van Bekkum, E. M. Flanigen, P. A. Jacobs, and J. C. Jansen (Elsevier, Amsterdam, 2001).

${ }^{2}$ R.M. Barrer and J.F. Cole, J. Phys. Chem. Solids 29, 1755 (1968).

${ }^{3}$ V. Srdanov, G.D. Stucky, E. Lippmaa, and G. Engelhardt, Phys. Rev. Lett. 80, 2449 (1998).

${ }^{4}$ G.K.H. Madsen, C. Gatti, B.B. Iversen, L. Damjanovic, G.D. Stucky, and V.I. Srdanov, Phys. Rev. B 59, 12359 (1999).

${ }^{5}$ I. Heinmaa, S. Vija, and E. Lipmaa, Chem. Phys. Lett. 327, 131 (2000).

${ }^{6}$ R. Windiks and J. Sauer, J. Chem. Phys. 113, 5466 (2000).

${ }^{7}$ O.F. Sankey, A.A. Demkov, and T. Lenosky, Phys. Rev. B 57, 15129 (1998).

${ }^{8}$ R. Windiks and J. Sauer, Phys. Chem. Chem. Phys. 1, 4505 (1999).
${ }^{9}$ B. Eisenstein, R. Prepost, and A.M. Sachs, Phys. Rev. 142, 217 (1966).

${ }^{10}$ A. Abragam, The Principles of Nuclear Magnetism (Oxford University Press, Oxford, 1961).

${ }^{11}$ R.E. Walstedt and L.R. Walker, Phys. Rev. B 9, 4857 (1974).

${ }^{12}$ MINUIT-Function Minimization and Error Analysis, CERN Program Library Entry D506, CERN, Geneva, Switzerland, 1996.

${ }^{13}$ R. Scheuermann, L. Schimmele, A. Seeger, T. Stammler, T. Grund, M. Hampele, D. Herlach, M. Iwanowski, J. Major, M. Notter, and T. Pfiz, Philos. Mag. B 72, 161 (1995).

${ }^{14}$ M. F. Collins, Magnetic Critical Scattering (Oxford University Press, New York, 1989).

${ }^{15}$ S. keng Ma, Modern Theory of Critical Phenomena (Addison Wesley, Boston, 1976).

${ }^{16}$ R. Kubo, Phys. Rev. 87, 568 (1952). 